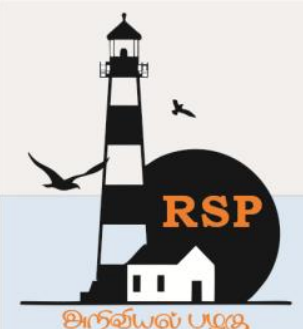
ADVANCED SCIENCE HUB

Special Issue of Second International Conference on Innovation in Engineering Sciences (ICIES2021)

\title{
The fourth order non oscillatory entropy stable scheme for degenerate convection diffusion equation part I
}

Jisha CR

Department of Mathematics, SRM Institute of Science and Technology, Kattankulathur-603203,

Tamil Nadu, India

jishacravi@gmail.com

\begin{abstract}
In this article, we formulate an entropy stable scheme for nonlinear degenerate convection diffusion equation with viscous terms in non-conservative formulations. Here in we extend the S.Jerez idea of first order entropy stable convection scheme for semi discrete scheme in to fourth order scheme using finite central difference scheme. Major advantage of this work is fourth order accuracy of the solution and fixed numerical diffusion term which can provide the non- oscillatory solution. Finally, few computational analyses are given to shown the accuracy of entropy stable scheme for degenerate parabolic equations.

Keywords: Entropy stable scheme, Hyperbolic conservation law, Convection diffusion equation, Central difference operator.

Mathematics Subject Classification Code: (2000) 60A10, 65K10, 49J20, 97N40, 60B10
\end{abstract}

\section{Introduction:}

In this section we discussed some basic definitions and results.

\subsection{Notations of difference operator}

We are discussing some general notations and basic formulas from [9]. The central difference with respect to $t$ are defined by

$\delta_{t_{0}}=\frac{w_{n}^{m+1}-w_{n}^{m-1}}{2}$

Similarly, with respect to $x$ is

$\delta_{x_{0}}=\frac{w_{n+1}^{m}-w_{n-1}^{m}}{2}$

The second central difference is defined by $\delta^{2}=\delta^{+} \delta^{-}$.

Here we have

$\delta_{x}^{2} w_{n}^{m}=\frac{w_{n+1}^{m}-2 w_{n}^{m}+w_{n-1}^{m}}{k^{2}}$
We need derive fourth order accurate aproximation to first and second derivative with respect to $x$

$$
\begin{aligned}
& \delta_{0} w=\frac{\partial w}{\partial x}+\frac{h^{2}}{6} \frac{\partial^{3} w}{\partial x^{3}}+O\left(h^{4}\right), \\
& =\left(1+\frac{h^{2}}{6} \frac{\partial^{2} w}{\partial x^{2}}\right) \frac{\partial w}{\partial x}+O\left(h^{4}\right), \\
& =\left(1+\frac{h^{2}}{6} \delta^{2}\right) \frac{\partial w}{\partial x}+O\left(h^{4}\right),
\end{aligned}
$$

Where,

$$
\frac{\partial^{2} w}{\partial x^{2}}=\delta^{2}+O(h)
$$

$\frac{\partial^{4} w}{\partial x^{4}}=\delta^{4}+O(h)$

Rewrite above equation (7) by 
$\frac{\partial w}{\partial x}=\left(1+\frac{h^{2}}{6} \delta^{2}\right)^{-1} \delta_{0} w+O\left(h^{4}\right)$

using the Binomial expansion

$\frac{\partial w}{\partial x}=\left(1-\frac{h^{2}}{6} \delta^{2}\right) \delta_{0} w+O\left(h^{4}\right)$,

Our main motivation is to introduced a fourth order non-oscillatory entropy stable scheme for non-degenerate convection diffusion equation. Next section 1.1 discussed the basic results of fourth order scheme. The present article is organized in the following way. Some preliminary results and definitions are given in sections 1.1 and 2 . The fourth order entropy stable scheme and new limiter are discussed in sections 3 and 3.1. Numerical results of proposed framework discussed in section 3.1 and 4 .

1.2 Forth order scheme for degenerate equation Let us consider the following equation (11) with non-conservative diffusion term in one Dimensional space.

$\mathrm{w}_{\mathrm{t}}+\mathrm{f}(\mathrm{w})_{\mathrm{x}}=\left(\mathrm{k}(\mathrm{w}) \mathrm{w}_{\mathrm{x}}\right)_{\mathrm{x}},(\mathrm{x}, \mathrm{t}) \in \mathrm{R} \times \mathrm{R}^{+}$

$\mathrm{w}(\mathrm{x}, 0)=\mathrm{w}_{0}(\mathrm{x})$.

Where fbe a nonlinear flux function of conserved vector quantity

$\mathrm{w}(\mathrm{x}, \mathrm{t}): \mathrm{R} \times \mathrm{R}^{+} \rightarrow \Omega \in \mathrm{R}^{\mathrm{n}}$ and $\mathrm{k}(\mathrm{w}) \in \mathrm{R}^{\mathrm{N} \times \mathrm{N}}$ is a positive semidefinite diffusion matrix defined in $\Omega$. The diffusion term vanish in some sub spatial interval. For simplicity we denote $w=w(x, t)$.

This type of system represented by in porous media flow and two phase flow model [1-5].

If $\frac{d K}{d w}=k$ then equation (11) will be the following conservative form,

$$
\mathrm{w}_{\mathrm{t}}+\mathrm{f}(\mathrm{w})_{\mathrm{x}}=\mathrm{K}(\mathrm{w})_{\mathrm{xx}},
$$

Due to this degeneracy of viscous term near shock may have solution in hyperbolic-parabolic dynamic problem.

Let us discretized the equation (11) using central difference operator,

$$
\frac{\partial f(w)}{\partial x}=\frac{\left(1+\frac{h^{2}}{6} \delta^{2}\right) \delta_{0} f(w)}{\partial x}+O\left(h^{4}\right),
$$

Using Taylor series expansion, approximate first derivative by fourth order approximation in $h$.

Similarly, $\frac{\partial k(w) w_{x}}{\partial x}=\frac{\partial\left(k\left(1+\frac{h^{2}}{6} \delta^{2}\right) \delta_{0}(w)(x)\right.}{\partial x}+O\left(h^{4}\right)$

By the product rule, R.H.S of the (14) is the following form. Let us consider $\frac{\partial k(w) w_{x}}{\partial x}=\frac{\partial(k L)}{\partial x}+O(h 4)$

L.H.S $=\frac{1}{2 h}\left(\frac{-1}{12}(K L)_{j+2}-(K L)_{j+2}\right)+$

$\frac{4}{3}(K L)_{j+1}-(K L)_{j-1}-\frac{5}{2}(K L)_{j}+O\left(h^{4}\right)$

$=\frac{1}{2 h}\left(\tilde{k}_{j+\frac{1}{2}}-\tilde{k}_{j-\frac{1}{2}}\right)+O\left(h^{4}\right)(15)$

where

$$
\begin{aligned}
& \tilde{k}_{j+\frac{1}{2}}=\frac{1}{h}\left(\frac{-1}{12} k_{j+2}\left(w_{j+2}-w_{j+1}\right)+\frac{7}{12} k_{j}\left(w_{j+1}-w_{j}\right)\right. \\
& +\frac{7}{12} k_{j}\left(w_{j-1}-w_{j-2}\right)-\frac{1}{12} k_{j-1}\left(w_{j-2}-w_{j-3}\right) \\
& =\frac{1}{h}\left(\frac{-1}{12} k_{j+2}[[w]]_{j+\frac{5}{2}}+\frac{7}{12} k_{j+1}[[w]]_{j+\frac{3}{2}}\right. \\
& +\frac{7}{12} k_{j}[[w]]_{j+\frac{1}{2}}-\frac{1}{12} k_{j-1}[[w]]_{j-\frac{1}{2}}-->(16)
\end{aligned}
$$

We replaced the value of $L$ by first order forward difference operator for representing the first derivative. Substitute the equation (16) in to (14), then we get a fourth order scheme. Replace the notation

$h=\Delta x$. The numerical scheme will be

$$
\begin{gathered}
\frac{\partial}{\partial t} w(x, t)=-\frac{\partial f(w(x, t))}{\partial x}+\frac{\partial}{\partial x} k(w(x, t)) \frac{\partial w(x, t)}{\partial x},(17) \\
=\frac{-1}{\Delta x}\left(F_{j+\frac{1}{2}}^{4}-F_{j-\frac{1}{2}}^{4}\right)+\frac{-1}{2 \Delta x^{2}}\left(\tilde{k}_{j+\frac{1}{2}}-\tilde{k}_{j-\frac{1}{2}}\right) \\
\widetilde{F}_{j+1 / 2}=\left(-\frac{1}{12} F_{j+2}+\frac{7}{12} F_{j+1}+\frac{7}{12} F_{j}\right. \\
\left.-\frac{1}{12} F_{j+1}\right)
\end{gathered}
$$

\section{Entropy stable scheme for degenerate convection diffusion equation}

\subsection{Basic results}

Let us consider (12) with $K \rightarrow 0$, then the equation have hyperbolic in nature. The main challenge for solving such type of equation is the shock wave formation near discontinuities of solution profile 


\section{www.rspsciencehub.com}

of nonlinear flux. Even for smooth initial data, the solution profile may have shock formation with nonlinear flux function. So we can use the weak formation in that situation. It is well known that weak solution do not converge to physically relevant unique solution. Lack of uniqueness of numerical solution must be enforced additional criteria, we call it as entropy condition. Next we discuss the entropy stability of the scheme (18).

The accuracy and efficiency of entropy stable methods and higher order of accuracy discussed in $[2,3,4]$ and discontinuous Galerkin methods in [5]. The entropy inequality for a degenerate parabolic problem Our main motivation of the study is to introduced the higher order entropy stable scheme. Entropy condition for system of equations (12) is defined in [6] Consider the three tuple ( $\eta, q, r)$ of functions from the set $\Omega$ to $R$, with strictly convex such that

$q_{w}^{T}=\eta_{w}^{T} f_{w}$

$r_{w}^{T}=\eta_{w}^{T} \frac{d k}{d w}$

$w^{T} \eta_{w w} \frac{d K}{d w} \geq 0, w \in \Omega$

where the subscript $w$ denotes the Hessian function. Recall that $\eta=\eta(w)$ and $q=q(w)$ are the entropy flux function, respectively, and the new function $r=r(w)$ is named the diffusion entropy flux. Since $\eta$ is strictly convex and $v=\eta_{w}$ is the entropy variable. Silvia Jerez Et.al. prove that degenerate form equation (12) is satisfy

$\eta(w)_{t}+q(w)_{x}-r(w)_{x x} \leq 0$,

The equation(12) said to be entropy stable if there exist two numerical entropy fluxes $Q_{j+1 / 2}$ and ${ }^{r_{j+1 / 2}^{\prime}}$ consistent with $q$ and $r_{x}$ which satisfies the following condition (see [6])

$\frac{1}{\Delta x}\left(r_{j+\frac{1}{2}}^{\prime}-r_{j-\frac{1}{2}}^{\prime}\right) \leq 0$

$$
\frac{d}{d t} \eta\left(w_{j}\right)(t)+\frac{1}{\Delta x}\left(Q_{j+\frac{1}{2}}-Q_{j-\frac{1}{2}}\right)-
$$

2.1.1. Entropy stable scheme with Entropy conservative flux

Let us consider the Lefloch formula for higher order flux,

$\tilde{F}_{j+1 / 2}^{2 p}=\sum_{r=1}^{p} \alpha_{r}^{p} \sum_{s=0}^{r-1} \widetilde{F(}\left(w_{j-s}, w_{j-s+r}\right)$
Volume 03 Issue 04S April 2021

Theorem 2.1. (see[ [3], Theorem 4.4]). For $p \in \mathrm{N}$, assume that $\alpha_{1}^{p}, \ldots, \alpha_{p}^{p}$ solve the $p$ linear equations

$$
\sum_{r=1}^{p} r \alpha_{r}^{p}=1, \sum_{i=1}^{p} i^{2 s-1}(s=2, \ldots p,)
$$

then $\widetilde{\mathrm{F}^{\{2 \mathrm{p}\}}}$ th-order accurate, in the sense that for sufficiently smooth solution $w$ and entropy conservative by following discrete entropy equality.

$\widetilde{Q}_{j+1 / 2}^{2 p}=\sum_{r=1}^{p} \alpha_{r}^{p} \sum_{s=0}^{r-1} \widetilde{Q}\left(w_{j-s}, w_{j-s+r}\right)$,

Substitute $p=2$ in entropy conservative flux

$$
\tilde{F}_{j+1 / 2}^{4}=\sum_{r=1}^{2} \alpha_{r}^{p} \sum_{s=0}^{r-1} \widetilde{F(}\left(w_{j-s}, w_{j-s+r}\right)
$$

and

$$
\widetilde{F_{j+\frac{1}{2}}}\left(w_{j}, w_{j+1}\right)=\frac{f\left(w_{j}\right)+f\left(w_{j+1}\right)}{2}
$$

Which imply that (28)

$\widetilde{F}_{j+1 / 2}=\left(-\frac{1}{12} F_{j+2}+\frac{7}{12} F_{j+1}+\frac{7}{12} F_{j}-\frac{1}{12} F_{j+1}\right)$,

The two-point finite difference scheme,

$\frac{\partial}{\partial t} w(x, t)=-\frac{1}{\Delta x}\left(\tilde{F}_{j+1 / 2}-\tilde{F}_{j+1 / 2}-\tilde{F}_{j-1 / 2}\right)+$

$\left.\left.\frac{1}{\Delta x^{2}}\left(k_{j+1 / 2}[\tilde{w}]\right]_{j+1 / 2}-k_{j-1 / 2}[\tilde{w}]\right]_{j-1 / 2}\right)$

is entropy stable provided the numerical viscosity matrix $k_{j+1 / 2}$ satisfies the following conditions

$\bar{v}_{j+1 / 2}^{T} k_{j+1 / 2}[w]_{j+\frac{1}{2}}=[r]_{j+1 / 2}$.

$\operatorname{And}[v]_{j+1 / 2}^{T} k_{j+1 / 2}[w]_{j+1 / 2} \geq 0$. (31)

proved in [6]. Here Jerez used entropy conservative flux, but due to the diffusion term numerical scheme become entropy stable provided $k=0$.Suppose if $k=0$ then scheme (29) will not be entropy stable. It satisfies only entropy conservative condition. In that case near discontinuity solution profile may exhibit Gibb's phenomenon near discontinuity region. To prevent this phenomenon, we need to add extra numerical diffusion term. Jerez modified the scheme for entropy stability that we discussed in next subsection. 
2.1.2. Entropy stable scheme with Entropy stable flux

Consider the fourth order entropy stable flux defined in [2]. If $K=0$ the numerical scheme equation will be

$\frac{\partial}{\partial t} w(x, t)=-\frac{1}{\Delta x}\left(F_{j+\frac{1}{2}}-F_{j-1 / 2),}(32)\right.$

replace $F_{j+1 / 2}$ by entropy stable flux $F_{j+1 / 2}^{4}$,

$F_{j+1 / 2}^{4}=\tilde{F}_{j+1 / 2}-\frac{1}{2} D_{j+\frac{1}{2}}[[v]]_{j+\frac{1}{2}}(33)$

Here $D$ is positive definite matrix. The scheme (32) using flux (33) is entropy stable by Cheng theorem 3.3 in [7] and results in [2].

Under the hypothesis of the theorem 3.2 in [6] the finite the entropy stability of the difference scheme,

$$
\begin{aligned}
& \frac{\partial}{\partial t} w(x, t)=-\frac{1}{\Delta x}\left(F_{j+\frac{1}{2}}-F_{j-\frac{1}{2}}\right)+ \\
& \frac{1}{\Delta x^{2}}\left(k_{j+\frac{1}{2}}[[w]]_{j+\frac{1}{2}}-k_{j-\frac{1}{2}}[[w]]_{j-\frac{1}{2}}\right)+ \\
& \frac{\varepsilon}{\Delta x^{2}}\left([[w]]_{j+\frac{1}{2}}-[[w]]_{j-\frac{1}{2}},\right.
\end{aligned}
$$

Where $\varepsilon>0$ is proved by Jerez.

\section{Fourth order entropy stable scheme for non-} degenerate convection diffusion equation

We consider fourth order entropy stable scheme for non-conservative formulation of diffusion term. For generating entropy stable fourth order scheme we need a central forth order scheme for convection diffusion equation with nonconservative diffusion term.

Substitute the flux (33) to the two point scheme (29) is modified to,

$$
\begin{aligned}
& \frac{\partial}{\partial t} w(x, t)=-\frac{1}{\Delta x}\left(F_{j+\frac{1}{2}}^{4}-F_{j-\frac{1}{2}}^{4}\right)+ \\
& \frac{v_{j}^{T}}{\Delta x^{2}}\left(\tilde{k}_{j+\frac{1}{2}}-\tilde{k}_{j-\frac{1}{2}}\right)(35)
\end{aligned}
$$

Remark 3.1. The entropy stable scheme (34) by Jerez we discussed, but in that scheme diffusion term not fixed for PDE, it may vary due to the varying. In this article we used sign stable fourth reconstruction for diffusion term. ie., sign $([[v]])=$ $\operatorname{sign}(<\langle v\rangle>)$ where

$\operatorname{sign}(x)=\left\{\begin{array}{l}1, \text { if } 0 \leq x \leq 1 \\ -1, \text { other wise }\end{array}\right.$

Theorem 3.1. The scheme (35) is entropy stable if it satisfies following condition. The numerical flux $F_{j+1 / 2}$ consistent with $f$ and satisfies $[v]_{j+1 / 2} F_{j+1 / 2}=[\varphi]_{j+1 / 2}$ and the numerical viscosity matrix $k_{j+1 / 2}$ verifies

$$
\begin{aligned}
& \tilde{v}_{j+\frac{1}{2}} k_{j+\frac{1}{2}}=\left[r^{\prime}\right]_{j+1 / 2}, \\
& \text { and } \\
& {[v]_{j+\frac{1}{2}}^{T} \tilde{k}_{j+1 / 2} \geq 0(38)}
\end{aligned}
$$

Proof. Multiply $v_{i}$ by both side of equation (12)

$\frac{d}{d t} \eta(w)_{j}=v_{j}^{T} \frac{1}{\Delta x}\left(\widetilde{F}_{j+\frac{1}{2}}-\tilde{F}_{j-\frac{1}{2}}\right)+\frac{v_{j}^{T}}{\Delta x^{2}}\left([[\widetilde{[k}]]_{j+\frac{1}{2}}-\right.$

$\left.[\widetilde{[k]}]_{j-\frac{1}{2}}\right)$

$v_{j}^{T} \frac{1}{\Delta x}\left(\tilde{F}_{j+\frac{1}{2}}-\tilde{F}_{j-\frac{1}{2}}\right)=\bar{v}_{j+1 / 2}^{T} \frac{1}{\Delta x} \tilde{F}_{j+\frac{1}{2}}-$

$\bar{v}_{j-\frac{1}{2}}^{T} \frac{1}{\Delta x} \tilde{F}_{j-\frac{1}{2}}-$

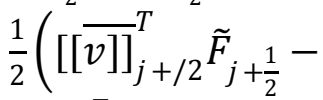

$\left[\left[{\bar{v}]]_{j-/ 2}^{T}}^{T} \tilde{F}_{j-\frac{1}{2}}\right)\right.$

The numerical flux $Q_{j+1 / 2}$ is defined by

$Q_{j+\frac{1}{2}}=q_{j+\frac{1}{2}}+\bar{v}_{j+\frac{1}{2}} F_{j+\frac{1}{2}}$

Similarly $v_{j}^{T} \bar{k}_{j+1 / 2}=v_{j}^{T}\left(\frac{-1}{12} k_{j+\frac{1}{2}}[[w]]_{j+\frac{3}{2}}-\right.$

$\frac{7}{12} k_{j+1[[w]]_{j+\frac{1}{2}}}+\frac{7}{12} k_{j-\frac{1}{2}}-\frac{1}{12} k_{j-1}[[w]]_{j-3 / 2}$

$v_{j}^{T} \tilde{k}_{j+\frac{1}{2}}=v_{j}^{T}\left(\frac{-1}{12} k_{j+2}[[w]]_{j+\frac{3}{2}}-\frac{7}{12} k_{j+1}[[w]]_{j+\frac{1}{2}}+\right.$

$\frac{7}{12} k_{j}[[w]]_{j-\frac{1}{2}}-\frac{1}{12} k_{j-1}[[w]]_{j-3 / 2}$,

$v_{j}^{T}\left(\tilde{k}_{j+\frac{1}{2}}-\tilde{k}_{j-\frac{1}{2}}\right)=\bar{v}_{j+\frac{1}{2}}^{T} \tilde{k}_{j+\frac{1}{2}}-\bar{v}_{j-\frac{1}{2}}^{T} \tilde{k}_{j-\frac{1}{2}}-$

$\frac{1}{2}\left([[v]]_{j+\frac{1}{2}} \tilde{k}_{j+\frac{1}{2}}-[[v]]_{j-\frac{1}{2}} \tilde{k}_{j-\frac{1}{2}}\right)$,

Substituting (43) and (41) in (39) then,

$$
\begin{aligned}
\frac{d}{d t} \eta\left(w_{j}\right)+\frac{1}{\Delta x} & \left(\tilde{Q}_{j+\frac{1}{2}}-\tilde{Q}_{j-\frac{1}{2}}\right) \\
& -\frac{1}{\Delta x}\left([[r]]_{j+\frac{1}{2}}-[[r]]_{j-\frac{1}{2}}\right) \\
& =-\frac{1}{2}\left[[[v]]_{j+\frac{1}{2}}^{T} \tilde{k}_{j+\frac{1}{2}}\right. \\
& -[[v]]_{j-1 / 2} \tilde{k}_{j-\frac{1}{2}} \leq 0
\end{aligned}
$$

After adding extra numerical diffusion term the equation (35) will be 


$$
\begin{aligned}
\frac{\partial}{\partial t} w(x, t)= & -\frac{1}{\triangle x}\left(F_{j+\frac{1}{2}}^{4}-F_{j-\frac{1}{2}}^{4}\right) \\
& +\frac{1}{\triangle x^{2}}\left(\tilde{k}_{j+\frac{1}{2}}-\tilde{k}_{j-\frac{1}{2}}\right)
\end{aligned}
$$

Where the flux $F_{j+\frac{1}{2}}^{4}=\tilde{F}_{j+\frac{1}{2}}-\frac{1}{2} D_{j+\frac{1}{2}}[[v]]_{j+\frac{1}{2}}$ is entropy stable flux

Theorem 3.2. A scheme with numerical flux equation (44) is entropy stable with numerical entropy flux given by

$q_{j+\frac{1}{2}}=\tilde{q}_{j+1 / 2}^{4}+\bar{v}_{j+1 / 2} D_{j+1 / 2}<<v>>_{j+1 / 2}$

is entropy stable with entropy flux is defined by

$Q_{j+\frac{1}{2}}^{4}=\tilde{Q}_{j+1 / 2}-\frac{1}{2} \bar{v}_{j+\frac{1}{2}} D_{j+\frac{1}{2}}[[v]]_{j+1 / 2}$

and $\dot{r}_{j+\frac{1}{2}}=\frac{1}{\Delta x} \bar{v}_{j+1 / 2} \tilde{k}_{j+1 / 2}$

with $\langle v\rangle_{j+\frac{1}{2}}=\beta_{j+\frac{1}{2}}[[v]]_{j+1 / 2}$, .

Existing tools for making higher order reconstruction is WENO-Z and JS-WENO e.t.c. But generally, it is not sign stable. Here we reconstruct.

$[[v]]_{j+1 / 2}$ by fifth order WENO-Z method[8], but it is not sign stable. So we introduced limiter for preserving the sign stable.

\subsection{Limiter for sign stability}

In this article for reconstructing $\langle v\rangle_{j-\frac{1}{2}}$ we used WENO-Z scheme, but it is not sign stable. So, we used by following way

$\phi(i)=\left\{\begin{array}{c}1, \text { if } 0 \leq s(j) \leq 1 \\ 0, \text { other wise }\end{array}\right.$

where $s(j)$ is the numerical jump at $x(j)$ ie.,

$s(i)=\frac{w_{j+1}-w_{j}}{v_{j+1}^{+} v_{j}^{-}}$

provided $v_{j+1}^{+}-v_{j}^{-} \neq 0$ otherwise $s(i)=0$.

Here we consider the general Courant-Friedrich-

Lewy(CFL) condition for parabolic equations is

$C F L=\max \left|f_{u}\right| \frac{\Delta t}{\Delta x}+2 \max |k| \frac{\Delta t}{\Delta x^{2}}<1$,

\section{Test problems}

Consider the equation

$w_{t}+\left(\frac{w^{2}}{2}\right)_{x}=K(w)_{x x},(x, t) \in[-2,2] \times[0, T]$

(56)

where $w \in[0, \infty]$ and diffusion matrix is defined as $K(w)=\mu w^{2}$, and $K^{0}(w)=k(w)$. Based on the known entropy flux pair $(\eta, g)$ for the

burger equation. Entropy 3-tuple $(\eta(w), g(w), r(w))$ $=\left(\frac{w^{2}}{2}, \frac{w^{3}}{3}, \frac{2}{3} \mu w^{3}\right)$ is satisfied equation 1.11 in [6], where $\mu=0.01$. Let us consider entropy stable scheme for that an entropy conservative numerical flux is consider. $F_{j+1 / 2}=\frac{1}{6}\left(w_{j+1}^{2}+w_{j+1} w_{j}+w_{j}{ }^{2}\right)$ and a numerical viscosity matrix satisfying theorem 3.4 in [6] by

$$
\begin{aligned}
& k_{j+1 / 2} \\
& =\left\{\begin{array}{c}
1, \quad \text { if } w_{j}, w_{j+1=0} \\
\mu \frac{w_{j}^{2}+w_{j} w_{j+1}+w_{j+1}^{2}}{w_{j}+w_{j+1}}, \quad \text { other wise }
\end{array}\right.
\end{aligned}
$$

Initial condition is $x \in[-2,2]$

$$
w_{0}(x)=\left\{\begin{array}{cc}
1, \quad \text { if }-0.5 \leq x \leq 0.5 \\
0, \quad \text { other wise }
\end{array}\right.
$$

For the computation we considering $N=1200$ for reference solution also. Mainly the following numerical methods are used for comparison

ES1 flux is entropy conservative, nonconservative discretization of source term, numerical scheme is 29. ES2 flux is entropy stable, non-conservative discretization of source term using the numerical scheme is 34. ES3 Fourth order entropy stable scheme. All test problem using ES3 are SSP-RK4 method used for solving differential equations. Numerical simulations of ES1 and ES2 are obtained combining an entropystable spatial discretization with a TVD-RK2 time stepping. The errors and convergence rate of ES1 and ES2 are shown in [6-9]. It is first order accurate. If $\mu \rightarrow 0$ the PDE have hyperbolic in nature. So spurious oscillation in solution profile will produce the numerical scheme near discontinuity. From figure 1 we can understand after adding extra diffusion, oscillation removed. Next we are discussing the numerical result for non-oscillatory higher order entropy stable scheme.

\section{Test problems for fourth order non- oscillatory entropy stable scheme}

Recalling the equation (12). If $\mu$ tends to zero in (58) tends to hyperbolic case. Entropy conservative methods capture appearance of propagation of shock wave correctly but may produce strong oscillations in near shock region. In order to reduce the oscillation in [6] add some viscosity term. This work useful to capture correctly the non-classical shocks due to parabolic and hyperbolic interaction. For detailed reference see [6]. 
www.rspsciencehub.com

$$
w_{t}+\left(\frac{w^{2}}{2}\right)_{x}=K(w)_{x x},(x, t) \in[-2,2] \times[0, T]
$$

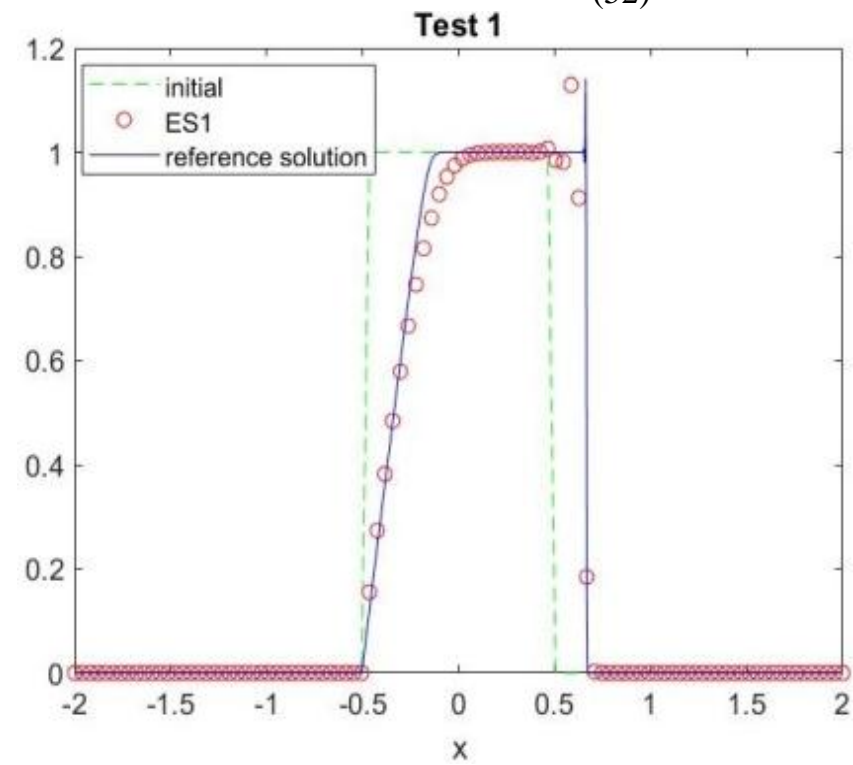

Figure 1: Solution for first order entropy conservative flux with 100 points with initial condition(51)

Example 5.1. [6] Let us consider the equation $w_{t}+\left(w^{2}\right)_{x}=\left(K(w) w_{x}\right)_{x},(x, t) \in[-1,1] \times[0,1], w \in \Omega$

where $\Omega=[0,+\infty]$.

with initial condition is defined by,

$\mathrm{w}_{0}(\mathrm{x})=\left\{\begin{array}{c}\left(1-\mathrm{x}^{2}\right)^{2}, \quad \text { if }-1 \leq \mathrm{x} \leq 1 \\ 0, \quad \text { other wise }\end{array}\right.$

,$x \in[-2,2]$. Entropy conservative numerical flux is defined by

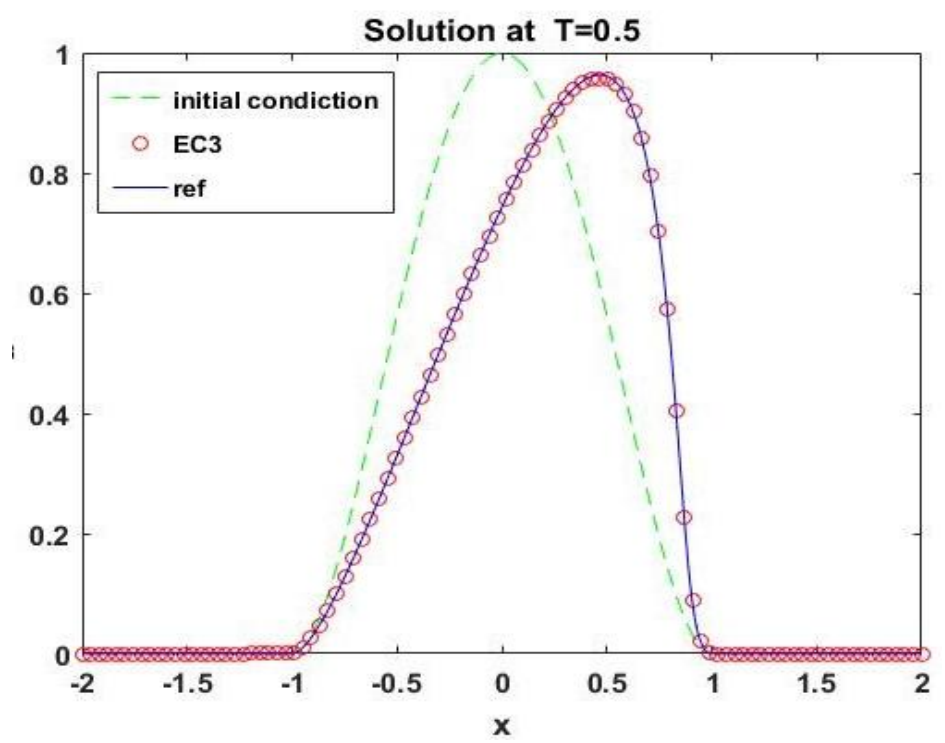

Figure 3: Numerical solution using ES3 with initial condition (54) and $\mathrm{N}=100, \mu=0.01, \mathrm{CFL}=0.9$
Volume 03 Issue 04S April 2021

where $w \in[0, \infty]$ and diffusion matrix is defined as $K(w)=\mu w^{2}$, and $K^{0}(w)=k(w)$.

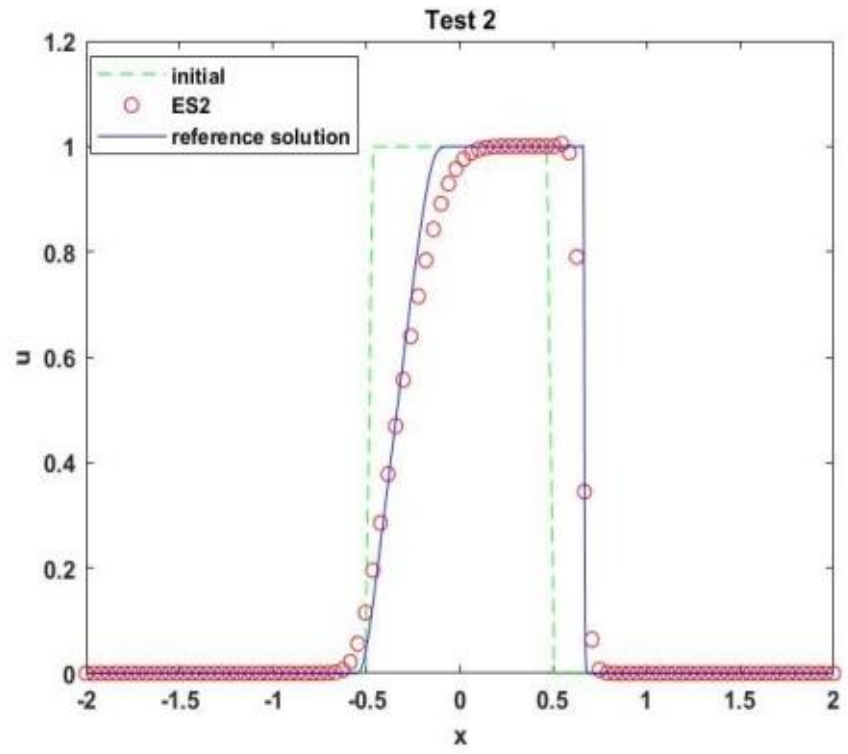

Figure 2: Solution for first order entropy stable flux with 100 points with initial condition(51)

$\mathrm{F}_{\mathrm{j}+\frac{1}{2}}^{4}=\widetilde{\mathrm{F}}_{\mathrm{j}+\frac{1}{2}}-\frac{1}{2} \mathrm{D}_{\mathrm{j}+\frac{1}{2}}[[\mathrm{v}]]_{\mathrm{j}+1 / 2}$

where ${ }^{D_{j+\frac{1}{2}} \text { is maximum of first derivative of } \mathrm{f} \text { and }}$ $\mathrm{v}$ is entropy variable. Numerical simulations of ES3 are obtained combining an entropystable spatial discretization with a TVD-RK4 time stepping. For that, we compute a reference solution with $\mu=0.1$ using the ES3 scheme with $\mathrm{N}=1200$ grid values.

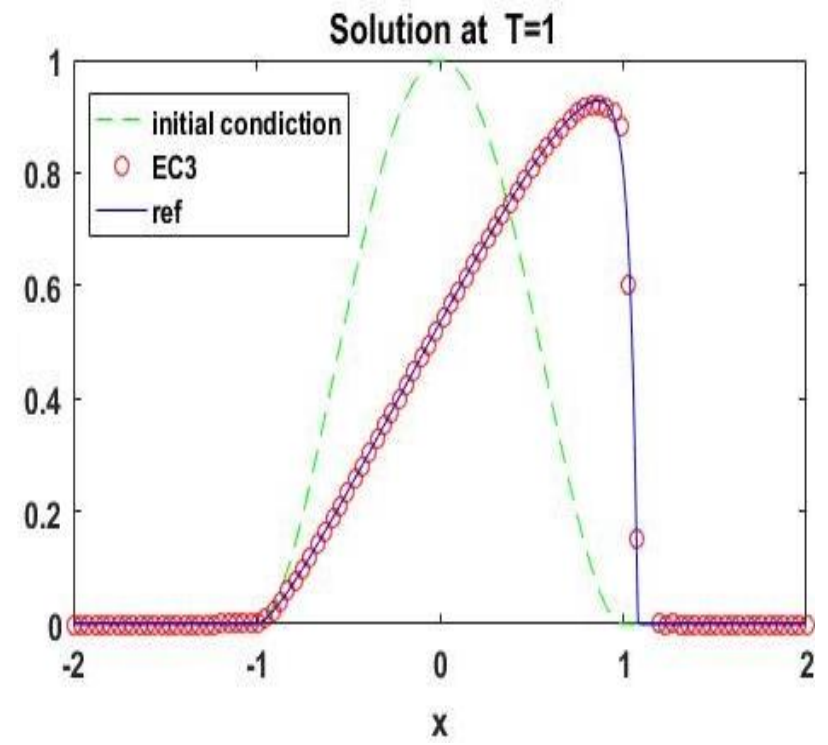

Figure 4: Numerical solution using ES3 with initial condition (60) and $\mathrm{N}=100, \mu=0.01$, $\mathrm{CFL}=0.9$ 
Example 5.2. Here we consider the equation

$w_{t}+\left(\frac{w^{2}}{2}\right)_{x}=K(w)_{x x},(x, t) \in[-2,2] \times$

$[0, T]$

with initial condition

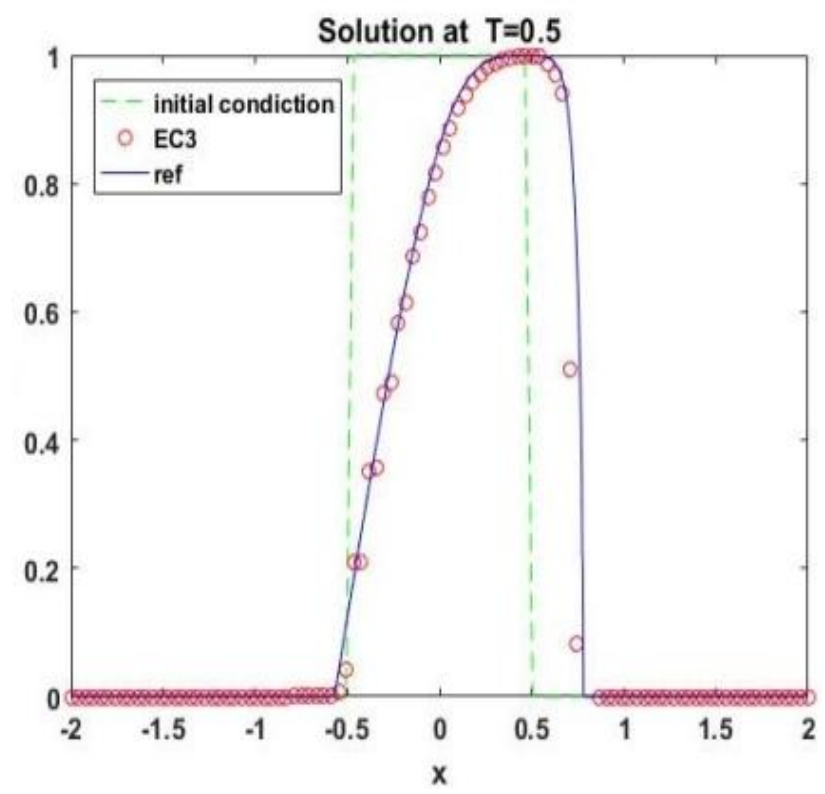

Figure 5: Numerical solution using ES3 with initial condition (54) and $\mathrm{N}=200, \mu=0.01, \mathrm{CFL}=0.5$

We used ES3 scheme with $\mathrm{CFL}=0.5$, and Final time $T=1$; Rate of convergence and accuracy table of the problem 57 is given.

\section{Conclusion}

Herein we introduce the concept of fourth order entropy stable scheme for degenerate convection equation and new limiter for preserving sign stability for numerical diffusion term. Using this limiter we can use all type of higher order WENO for reconstruction of diffusion term, Because of this we can generate a higher order nonoscillatory entropy stable scheme.

\section{References}

[1] Raimund, B., Coronel, A. I., and Mauricio, S., (2006)On an upwind difference scheme for strongly degenerate parabolic equations modelling the settling of suspensions in centrifuges and non-cylindrical vessels. Applied numerical mathematics, 56, 13971417.

[2] Fjordholm, U. S., Mishra, S., and Tadmor, E.,(2012) Arbitrarily high-order accurate entropy stable essentially non-oscillatory

$$
\begin{aligned}
& w_{0}(x) \\
& =\left\{\begin{array}{c}
1, \quad \text { if }-0.5 \leq x \leq 0.5 \\
0, \quad \text { other wise }
\end{array}\right.
\end{aligned}
$$

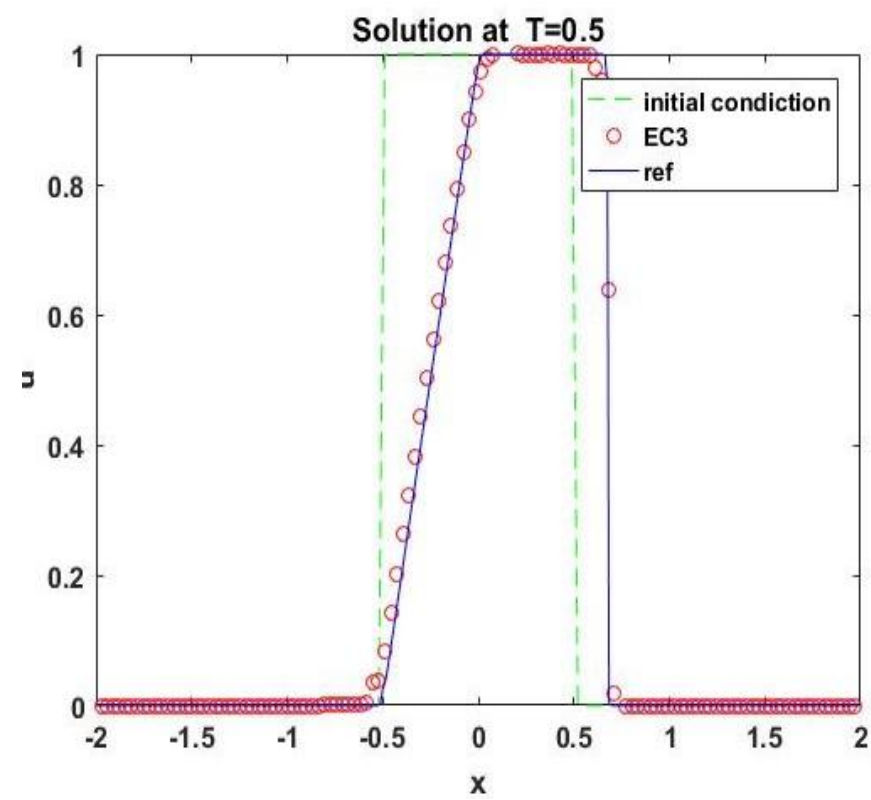

Figure 6: Numerical solution using ES3 with $\mathrm{N}=200, \mu=0.00, \mathrm{CFL}=0$.

schemes for systems of conservation laws. SIAM Journal on Numerical Analysis, 50(2), 544-573.

[3] Lefloch, G. P., Mercier, J. M., and Rohde, C.,(2002) Fully discrete, entropy conservative schemes of arbitraryorder. SIAM Journal on Numerical Analysis, 40(5), 1968- 1992.

[4] Liu, Y., Shu, C. W., and Zhang, M., (2011) High order finite difference weno schemes for nonlinear degenerate parabolic equations. SIAM Journal on Scientific Computing, 33(2), 939-965.

[5] Hiltebrand, A., and Mishra, S.,(2014) Entropy stable shock capturing space-time discontinuous galerkin schemes for systems of conservation laws. NumerischeMathematik, 126(1), 103-151.

[6] Jerez,S., and Pares, C., (2017) Entropy stable schemes for degenerate convection-diffusion equations. SIAM Journal on Numerical Analysis, 55(1), 240-264.

[7] Cheng, X., and Nie, Y., (2016) A third-order entropy stable scheme for hyperbolic 
Conservation laws. Journal of Hyperbolic Differential Equations, 13(01), 129-145.

[8] Castro, M., Costa, B., and Don, W. S.,(2011) High order weighted essentially nonoscillatoryweno-z schemes for hyperbolic conservation laws. Journal of Computational Physics, 230(5), 1766-1792.

[9] Strikwerda, John C. Finite difference schemes and partial differential equations. Society for Industrial and Applied Mathematics, 2004. 